\title{
A 3D cone beam computed tomography study of the styloid process of the temporal bone
}

\author{
F. Andrei ${ }^{1}$, A.G.M. Motoc ${ }^{2}$, A.C. Didilescu' ${ }^{3}$, M.C. Rusu 4 \\ 1 "Victor Babeş" University of Medicine and Pharmacy, Timişoara, Romania \\ 2Department of Anatomy and Embryology, Faculty of Medicine, "Victor Babeş" University of Medicine \\ and Pharmacy, Timişoara, Romania \\ ${ }^{3}$ Discipline of Embryology, Faculty of Dental Medicine, "Carol Davila" University of Medicine and Pharmacy, \\ Bucharest, Romania \\ "Discipline of Anatomy, Faculty of Dental Medicine, "Carol Davila" University of Medicine and Pharmacy, \\ Bucharest, Romania
}

[Received 19 August 2012; Accepted 28 November 2012]

Background: To investigate the length and three-dimensional orientation and to detail the morphological variations of the styloid process.

Materials and methods: Forty-four patients undergoing temporal bone evaluation for different reasons were randomly selected and included in the present study. The length, angulation in the coronal and sagittal planes, as well as morphological variations of the styloid processes were assessed using conebeam computer tomography. Pearson's correlation coefficient was used to test possible associations between the length of styloid process and angulations, as well as between angulations. Student's t-test was used to compare the differences between the sample mean length and angulations in normal and elongated styloid process groups.

Results: The sagittal angle showed weak positive correlations with the styloid process length and the transverse angle $(r=0.24, p=0.02, n=88)$. A medium positive correlation was found between the sagittal and transverse angulations in the elongated styloid process group ( $r=0.49, p=0.0015, n=38$ ). There was a statistical significant difference between the mean sagittal angulation in elongated styloid and normal styloid process groups $(p=0.015)$. The styloid process morphology also varied in terms of shape, number, and degree of ossification.

Conclusions: The morphometric and morphologic variations of the styloid process may be important factors to be taken into account not only from the viewpoint of styloid syndromes, but also in preoperatory planning and during surgery. (Folia Morphol 2013; 72, 1: 29-35)

Key words: branchial region, cone-beam computed tomography, elongated styloid process syndrome, glossopharyngeal nerve injuries, ossification

\section{INTRODUCTION}

The styloid chain derives from the Reichert's cartilage of the second branchial (pharyngeal) arch, which consists of four parts: tympanohyal, stylohyal, ceratohyal, and hypohyal [15]. The postnatal styloid chain consists of the styloid process of the

Address for correspondence: M.C. Rusu, MD, PhD, University of Medicine and Pharmacy "Carol Davila", 8 Eroilor Sanitari Blvd., RO-76241, Bucharest, Romania, tel: +40722363705, e-mail: anatomon@gmail.com 
temporal bone, the stylohyoid ligament, and the lesser horn of the hyoid bone [23], which correspond to all the parts of the cartilage of Reichert. Ossification of the styloid chain may determine a number of individual segments, separated by pseudoartroses [23].

Elongated styloid process (SP) gives a complex of symptoms described by Eagle in 1937, hence the condition is also known as Eagle's syndrome; symptoms are secondary to calcification of the stylohyoid ligament or to an elongated SP [2, 25]. Normal length of styloid process was stated by Eagle as $2.5 \mathrm{~cm}$ [14]. There are authors that take normal values up to $3 \mathrm{~cm}$ for the SP length $[8,17,22]$.

Symptoms of Eagle's syndrome include neck, throat, and hemifacial pain; sensation of foreign body in the throat; dysphagia; otalgia; and change in voice $[2,10,11,14]$. In addition to careful clinical examination, the use of plain radiographs, orthopantomograms, or computed tomography (CT) scan allows accurate preoperative diagnosis of this syndrome. Surgical treatment positively resolves the symptoms [6]. The transcervical or intraoral surgical approach to resection in patients with elongated SPs and Eagle syndrome appears to be safe and effective $[10,11,18]$.

The aim of the study was to investigate the length and three-dimensional (3D) orientation, and their possible associations with gender and aging, as well as to detail the morphological variations of SPs, using cone bean computer tomography (CBCT). No association between angulation, age, gender, and SP length defined the null hypothesis formulated in the present study.

\section{MATERIALS AND METHODS}

Forty-four patients ( 16 males), mean age of 42.18 \pm 13.44 years (range $21-63$ ), undergoing skull base evaluation for dental purposes were randomly selected and were included in this retrospective study. In the present study none of the subjects declared a previous history suggestive of Eagle syndrome.

All subjects were scanned using a CBCT machine - iCat (Imaging Sciences International) - and the CT data were analysed using dedicated software iCatVision. All studies were obtained using the same scanning protocol: sensor dimension: $20 \times 25 \mathrm{~cm}$; grayscale resolution: $14 \mathrm{bit}$; voxel dimension: 0.25 $\mathrm{mm}$; acquisition time: $13.9 \mathrm{~s}$; $120 \mathrm{KV}, 5 \mathrm{~mA}$; number of acquired images: 528 .

We used the iCAT Vision viewing Software for measurements, then the patients' files were transferred to the three-dimensional volume-rendering
(3DVR) program which was used to explore the 3D volume renderisations, and to document the cases in different view types (bone, TMJ, sinus/bone, or transparent skin).

All images were examined, according to the same protocol, by each of the authors of the present study, then the individual results were discussed and a general version was established.

The length of each SP was measured from its insertion site to the petrous part of the temporal bone to its tip, no matter the ossification degree and number of segments (1-3) the SP had.

Angulation of the SP was assessed in the coronal and sagittal planes, respectively. The medial angle between the long axis of the SP and a line connecting the bases of both SPs was defined as the transverse angle. The anterior angle between the long axis of the SP and the skull base was defined as the sagittal angle.

Cases were selected to be discussed from the point of view of peculiar morphologies and CT diagnostics.

\section{Statistical analysis}

The length and the angles were recorded separately for each side since there were some differences between the right and left. Data distributions were expressed as means, standard deviations (SD), and ranges. Pearson's correlation coefficient was used to test possible associations between the length of SP and angulations, as well as between angulations. Cluster analysis was performed to explore possible groups in data. Student's $t$-test and one-way ANOVA test were used to compare the differences between the sample mean length and angulations in various stratifications. Linear regression was used to test if the SP length was influenced by aging. All tests of significance were two tailed.

The data were analysed statistically on the computer using StatalC 11 (StataCorp. 2009. Stata: Release 11. Statistical Software. College Station, TX: StataCorp LP). A p-value $<0.05$ was considered statistically significant.

\section{RESULTS}

Overall, the results of measurements are shown in Table 1. The sagittal angle showed weak positive correlations with the SP length and the transverse angle $(r=0.24, p=0.02, n=88)$. No statistically significant differences were recorded between the evaluations performed on left and right sides. Gender did not influence the length or sagittal angula- 
Table 1. Mean length and transverse sagittal angles of styloid processes in the study group $(\mathrm{n}=88)$

\begin{tabular}{lcc}
\hline Variable & Mean (SD) & Range \\
\hline Styloid process length $[\mathrm{mm}]$ & $35.09(8.9)$ & $20.94-56.81$ \\
Transverse angulation $\left[^{\circ}\right]$ & $66.74(6.35)$ & $52.1-85.6$ \\
Sagittal angulation $\left[^{0}\right]$ & $53.98(8.16)$ & $29.7-69.1$ \\
\hline
\end{tabular}

Table 2. Descriptive analysis of the three groups obtained by complete linkage clustering

\begin{tabular}{ccccc}
\hline Group & N & $\begin{array}{c}\text { Styloid process length } \\
\text { (SD) [mm] }\end{array}$ & $\begin{array}{c}\text { Transverse angulation } \\
\text { (SD) [0] }\end{array}$ & $\begin{array}{c}\text { Sagittal angulation } \\
\text { (SD) [0] }\end{array}$ \\
\hline 1 & 18 & $48.82(3.65)$ & $68.51(4.86)$ & $58.58(6.23)$ \\
2 & 38 & $36.22(3.3)$ & $65.75(7.07)$ & $53.72(9.11)$ \\
3 & 32 & $26.02(2.48)$ & $66.92(6.12)$ & $51.67(6.97)$ \\
\hline
\end{tabular}

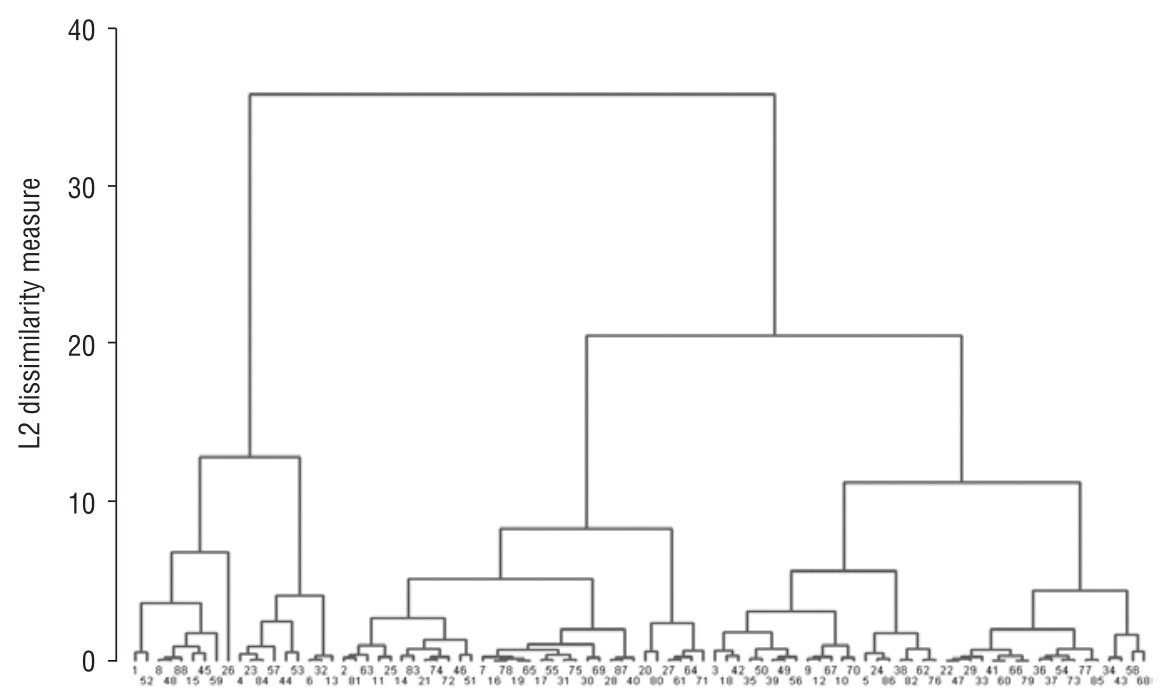

Figure 1. Dendrogram using complete linkage.

tion of SP. However, the difference between the mean transverse angulation in males and females was $3.49^{\circ}$, with a $95 \%$ confidence interval from 0.78 to 6.2 ; the t-test statistic was 2.56 , with $86^{\circ}$ of freedom and an associated $p$-value of 0.012 . The length of SP was not influenced significantly by aging.

\section{Cluster analysis}

Complete linkage clustering was chosen for this type of analysis. Three groups of SPs were suggested (Table 2, Fig. 1). The mean age did not vary significantly between the three groups. No asso- ciation was found between gender and groups. Statistically significant differences were recorded between the SP lengths of the three groups ( $p<$ $<0.0001$ ).

Sagittal angulations displayed statistically significant differences between the three groups ( $p=$ $=0.013$ ). The first group had higher values of angulations comparing with the third group ( $p=0.014$, Scheffe correction). Although the values of transverse angulations were slightly higher in the first group, they did not differ significantly when compared with the other two groups. 


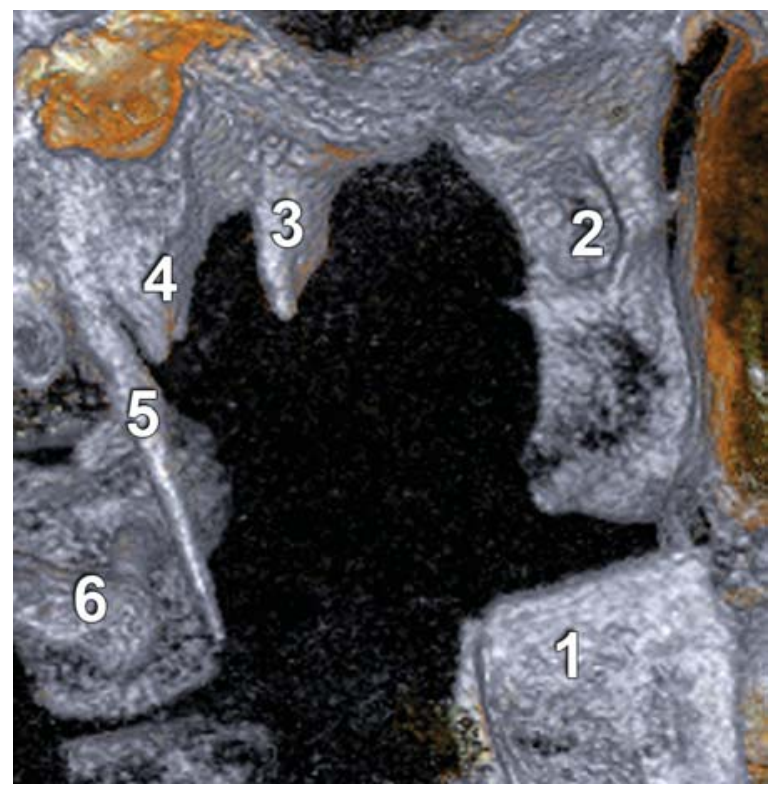

Figure 2. Three-dimensional volume rendering (bone/sinus view mode) lateral view of a right styloid process (SP); 1 - mandibular branch; 2 - pterygoid process; 3 - sphenoidal spine; 4 sheath of the SP; 5 -SP; 6 - transverse process of atlas.

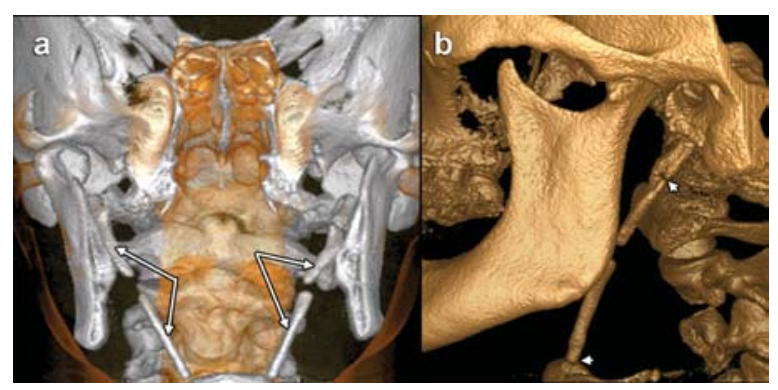

Figure 3. Bilateral, completely ossified styloid chains (arrows) (bilateral surgical resections); A. Anterior view, three-dimensional volume rendering (3DVR), bone/sinus view mode; $\mathbf{B}$. Left lateral view, 3DVR, TMJ view mode, two pseudoartroses (arrowheads) join the ossified segments of the styloid chain.

\section{The morphology of the styloid process}

The morphology of the SP and related bony landmarks were evaluated on 3DVRs (Fig. 2), and additional details were collected on multiplanar reconstructions (MPRs). Morphological variations were recorded (Figs. 3-10), and classified as follows:

1. length variations:

a. normal length (groups 2 and 3)

b. elongated SP (group 1) (Fig. 3)

2. variations of angulation

3. shape variations: linear (Fig. 2), scalariform (Fig. 5), or moniliform (Fig. 6), or pseudoduplicate shapes of the SP (Fig. 10)

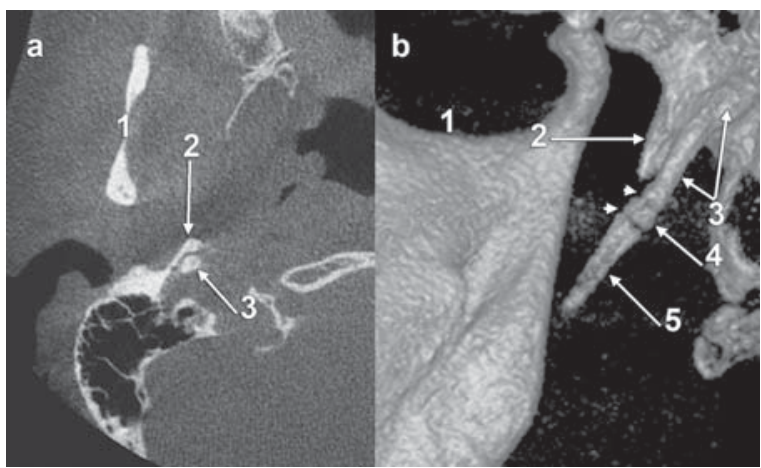

Figure 4. False duplicate styloid process; A. Axial multiplanar reconstructions; B. Three-dimensional volume rendering (bone view mode), medial view, right side; 1 - mandibular branch; 2 - elongated process of the tympanic part of the sheath of the styloid process (SP); 3 - long tympanohyal segment of SP; 4 short stylohyal segment of SP; 5 - ceratohyal segment of SP. The segments of the SP are separated by pseudoartroses (arrowheads).

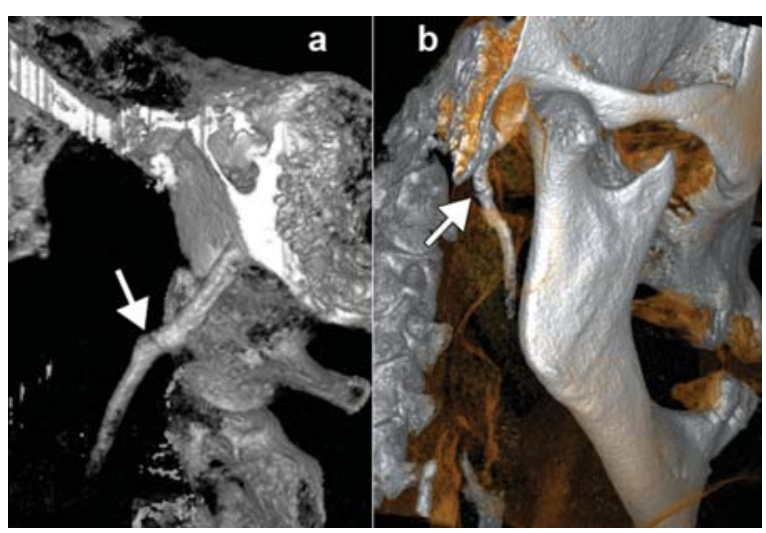

Figure 5. Bilateral scalariform styloid process, consisting of two pieces joined by a pseudoartrosis (arrows); $A$. Lateral view, three-dimensional volume rendering (3DVR), bone view mode; B. Postero-infero-lateral view, 3DVR, transparent skin view mode.

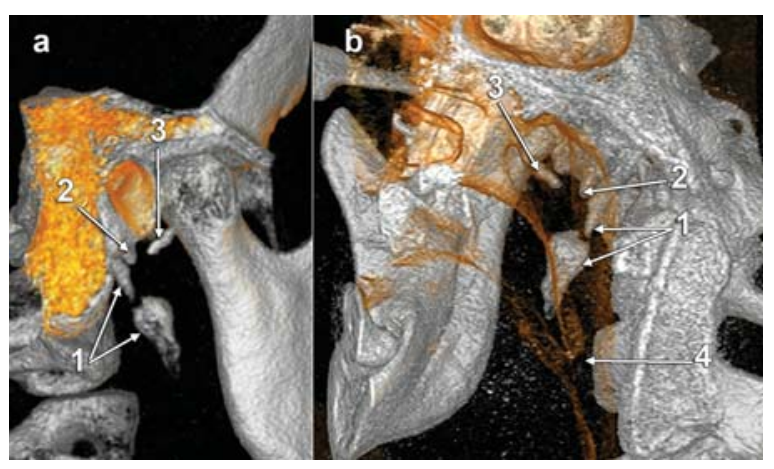

Figure 6. Right bipartite moniliform styloid process (SP), with incomplete ossification; A. Postero-lateral view, three-dimensional volume rendering (3DVR), sinus/bone view mode; $\mathbf{B}$. Medial view, 3DVR, transparent skin view mode; 1 -SP, consisting of two parts (stylohyal and ceratohyal); 2 - sheath of SP (false styloid); 3 - calcification within the inferior wall of the cartilaginous external auditory meatus; 4 - pharyngeal cavity. 


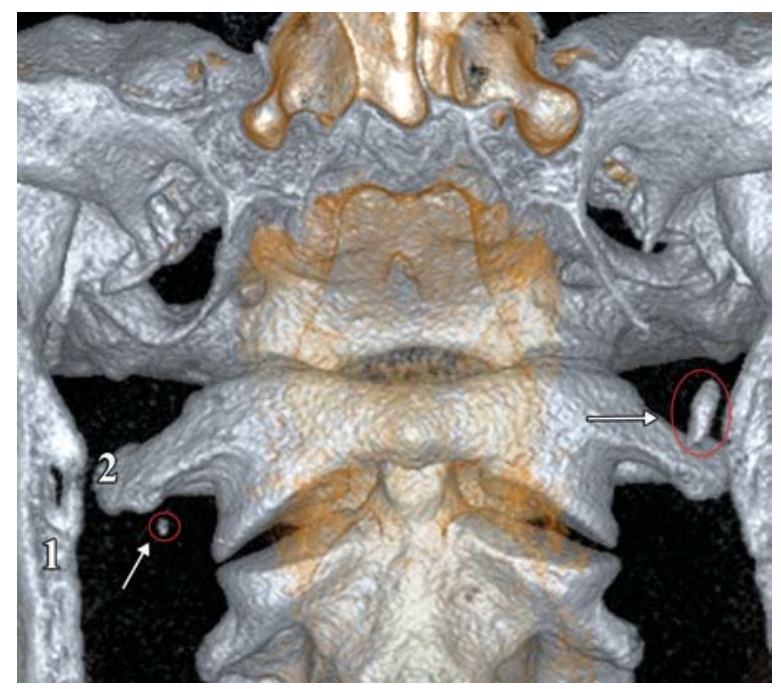

Figure 7. Bilateral almost complete lack of ossification of the styloid processes (red shapes), anterior view (3DVR, transparent skin view mode). The segments of the styloid chain cannot be evaluated; 1 - mandibular branch; 2 - transverse process of atlas.

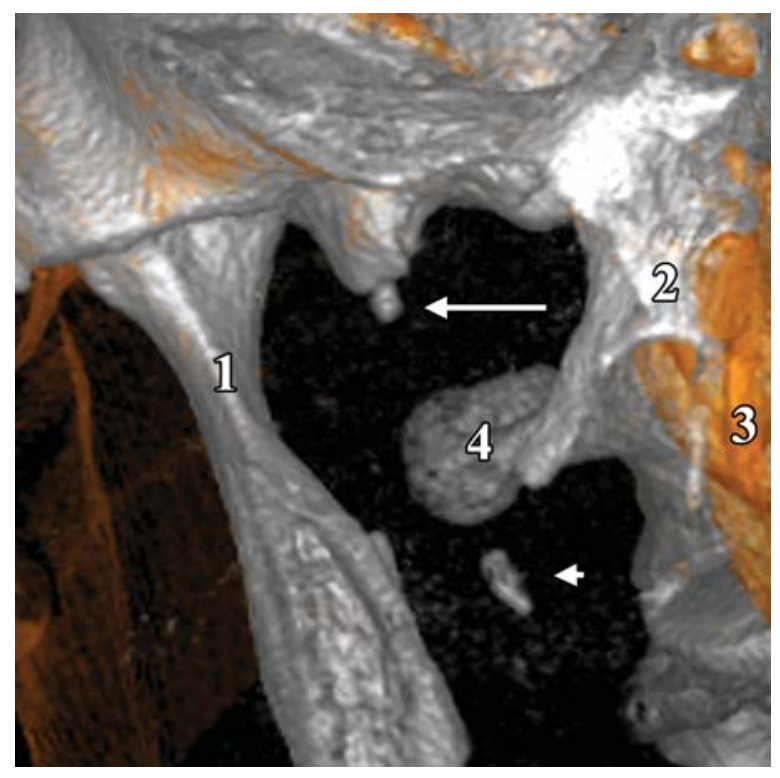

Figure 8. Antero-lateral view (3DVR, transparent skin view mode) of the right styloid process; only the tip of the tympanohyal segment (arrow) and a small part of the ceratohyal segment (arrowhead) are ossified; 1 - mandibular condyle; 2 - pterygoid process; 3 - pharyngeal cavity; 4 - transverse process of atlas.

4. variations of the number of pieces, separated by pseudoartroses (if more than one):

a. one piece:

i. SP proper: fused tympano- and stylohyal

ii. tympanohyal

iii. fused SP proper and partly ossified ceratohyal (Fig. 2)

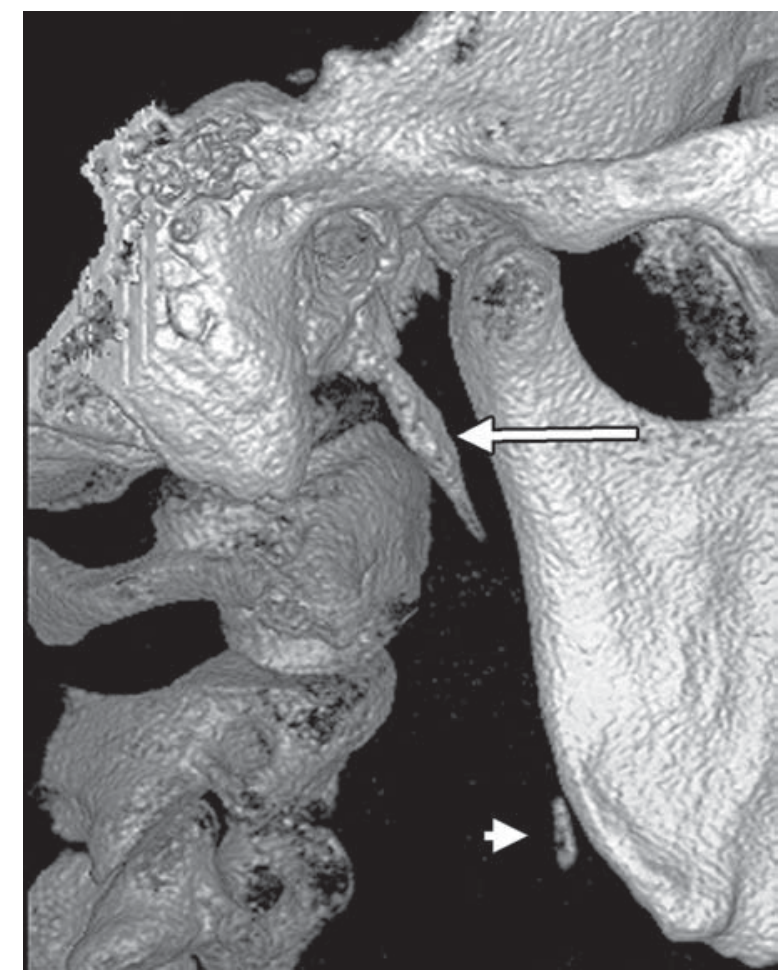

Figure 9. Outer view of the right styloid process (3DVR, bone view mode). The stylohyal (arrow) and a small ossification point (arrowhead) of the ceratohyal are indicated.

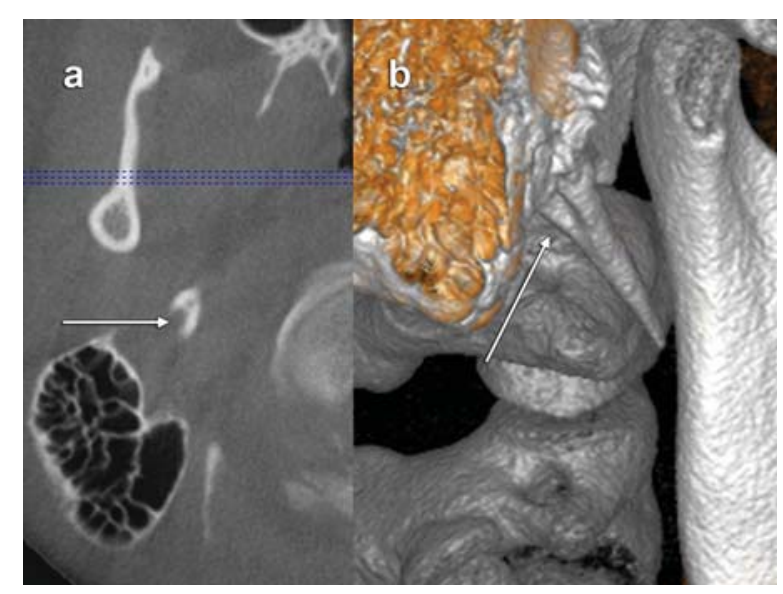

Figure 10. Pseudoduplicated right styloid process (arrow);

A. Axial multiplanar reconstructions; B. Three-dimensional volume rendering, postero-lateral view, sinus/bone view mode. 
d. other variants of multiple segments

5. variable degree of ossification:

a. complete ossification:

i. SP proper

ii. completely ossified styloid chain (Fig. 3)

b. incomplete ossification, in various combinations (Figs. 6-9)

\section{DISCUSSION}

The present study aimed to analyse the SP parameters that might influence the occurrence of styloid syndromes. It has been previously proven that 3D CT is an effective method in the evaluation of SP length, angulation, and other morphological characteristics [3, 24]. However, the usual CT tools, such as MPRs, should not be ignored, but used and presented complementarily. Using only 3D CT tools could lead to misdiagnoses (e.g. false SP: a prominent angle of the SP sheath can be easily confused with a duplicated SP [3]).

Measurements performed by different methods revealed that the mean length of SP may vary within an interval larger than $1 \mathrm{~cm}[1,9,13,21]$. Usually, an $\mathrm{SP}$ measuring $2.5 \mathrm{~cm}$ in length is considered to be normal, but if its length exceeds $3 \mathrm{~cm}$, the SP is regarded as elongated $[4,5,21]$. In our study, the SP length was high, the prevalence of the SPs with length $>35 \mathrm{~mm}$ being above $40 \%$. This may be explained by local population particularities. It is generally admitted that processes longer than $30 \mathrm{~mm}$ may induce symptoms that are classically reported in styloid syndromes. On the other hand, the contrast between high anatomic occurrence of long SP and low clinical manifestation was previously mentioned [16]. Not only the length of the SP is a pathogenic factor involved in occurrence of styloid syndromes, but also the shape and incurvation may be important.

It has been suggested that only long SPs directed downward, ventrally, and medially may lead to a styloid syndrome [16]. Conversely, there might be a lack of neurovascular damage in situations in which a long SP is partly ossified. Minor ossifications along the styloid chain, such as those we encountered, are able to determine minor clinical syndromes that may be misdiagnosed if a routine $\mathrm{CT}$ is not performed.

The transverse angulation ranged from $52.1^{\circ}$ to $85.6^{\circ}$, comparable with other studies $[3,14,19]$. It was proposed that a narrow angle of SP $\left(<65^{\circ}\right)$ may imply some complaints because of compression of adjacent structures [3]. Thirty-one SPs were found with narrow angulations in the present study. Among them, 21 belonged to the group of normal length SPs. In
Eagle's syndrome, a medial deviation of the SP becomes symptomatic by irritating the outside of the lateral pharyngeal wall [12].

The mean sagittal angle was much lower than previously reported [19]. This may be due to different landmarks used for evaluation. An elongated SP associated with a narrow sagittal anterior angle may irritate some structures coursing through the parapharyngeal space, e.g. the glossopharyngeal nerve. However, in the present study, a weak positive correlation was found between the SP length and the sagittal angle, suggesting that the role of sagittal incurvation is less important in this pathology.

No correlation was found between transverse angulation and SP's length, in agreement with previous reported results [3]. Conversely, there was a medium positive correlation between transverse and sagittal angulation in the elongated SP group, suggesting that a long SP is more prone to affect the elements coursing the retrostyloid space, the vessels (internal carotid, internal jugular) and the glossopharyngeal nerve being more exposed.

Anatomical variations of the SP and related structures, that is: elongated, segmented, pseudoarticulated, bent, partially ossified, absent (total or proximal part), double proximal part, and ossification of the stylohyoid ligament, have been described [3, 9, 23]. Such variations (see the classification in the Results section) are important to be taken into account not only from the viewpoint of styloid syndromes, but also in preoperatory planning and during surgery.

Eagle's syndrome (elongated SP syndrome) is a rare disease and $1.5-3.0 \%$ of adults have some of the complaints due to this pathology [7]. Eagle's syndrome develops due to an elongation or deformation of the SP or ossification/calcification of the SP [7, 20, 22]. In our opinion, a CBCT study, such as the present one, cannot evaluate whether the styloid elongation is due either to a developmental anomaly or to a ligament ossification.

\section{CONCLUSIONS}

The present study indicates that an increase of the sagittal angle is associated with an increase in SP length. The mean transverse angulation was higher in males than in females. With respect to these results, the null hypothesis was rejected. Although gender, aging, and transverse angulation did not influence the SP length, further studies on larger population samples should investigate possible variations that might occur. The morphological classification that was provided here establishes an important concept of morphological diversity of the SP of the temporal bone. 


\section{ACKNOWLEDGEMENTS}

We would like to thank Dr. Mihai Sandulescu, Discipline of Oral Implantology, "Carol Davila" University of Medicine and Pharmacy, Bucharest for his help in data collecting. All authors contributed equally to this study.

\section{REFERENCES}

1. Bafaqeeh SA (2000) Eagle syndrome: classic and carotid artery types. J Otolaryngol, 29: 88-94.

2. Balbuena L, Jr., Hayes D, Ramirez SG, Johnson R (1997) Eagle's syndrome (elongated styloid process). South Med J, 90: 331-334.

3. Basekim CC, Mutlu H, Gungor A, Silit E, Pekkafali Z, Kutlay M, Colak A, Oztürk E, Kizilkaya E (2005) Evaluation of styloid process by three-dimensional computed tomography. Eur Radiol, 15: 134-139.

4. Eagle WW (1958) Elongated styloid process; symptoms and treatment. A.M.A. Archives Otolaryngol, 67: 172-176.

5. Eagle WW (1962) The symptoms, diagnosis and treatment of the elongated styloid process. Am Surg, 28: 1-5.

6. Fini G, Gasparini G, Filippini F, Becelli R, Marcotullio D (2000) The long styloid process syndrome or Eagle's syndrome. J Craniofac Surg, 28: 123-127.

7. Gervickas A, Kubilius R, Sabalys G (2004) Clinic, diagnostics and treatment pecularities of Eagle's syndrome. Stomatologija, Baltic Dental Maxillofacial J, 6: 11-13.

8. Gokce C, Sisman Y, Sipahioglu M (2008) Styloid process elongation or eagle's syndrome: is there any role for ectopic calcification? Eur J Dentistry, 2: 224-228.

9. Gozil R, Yener N, Calguner E, Arac M, Tunc E, Bahcelioglu M (2001) Morphological characteristics of styloid process evaluated by computerized axial tomography. Ann Anat, 183: 527-535.

10. Hossein R, Kambiz M, Mohammad D, Mina N (2010) Complete recovery after an intraoral approach for Eagle syndrome. J Craniofac Surg, 21: 275-276.

11. Jackson FE (1974) Hemicrania secondary to elongated styloid process "the Eagle Syndrome". Diseases Nervous System, 35: 528-531.

12. Kaufman SM, Elzay RP, Irish EF (1970) Styloid process variation. Radiologic and clinical study. Arch Otolaryngol, 91: 460-463.
13. Keur JJ, Campbell JP, Mccarthy JF, Ralph WJ (1986) The clinical significance of the elongated styloid process. Oral Surg Oral Med Oral Pathol, 61: 399-404.

14. Kosar Ml, Atalar MH, Sabanclogullari V, Tetiker H, Erdil FH, Cimen M, Otag I (2011) Evaluation of the length and angulation of the styloid process in the patient with prediagnosis of Eagle syndrome. Folia Morphol, 70: 295-299.

15. Krennmair G, Lenglinger F, Lugmayr H (2001) Variants of ossification of the stylohyoid chain. RoFo: Fortschritte auf dem Gebiete der Rontgenstrahlen und der Nuklearmedizin, 173: 200-204.

16. Lengele BG, Dhem AJ (1988) Length of the styloid process of the temporal bone. Arch Otolaryngol Head Neck Surg, 114: 1003-1006.

17. Lorman JG, Biggs JR (1983) The Eagle syndrome. Am J Roentgenol, 140: 881-882.

18. Martin TJ, Friedland DR, Merati AL (2008) Transcervical resection of the styloid process in Eagle syndrome. Ear Nose Throat J, 87: 399-401.

19. Onbas O, Kantarci M, Murat Karasen R, Durur I, Cinar Basekim C, Alper F, Okur A (2005) Angulation, length, and morphology of the styloid process of the temporal bone analyzed by multidetector computed tomography. Acta Radiol, 46: 881-886.

20. Orhan KS, Guldiken Y, Ural HI, Cakmak A (2005) Elongated styloid process (Eagle's syndrome): literature review and a case report. Agri, 17: 23-25.

21. Palesy P, Murray GM, De Boever J, Klineberg I (2000) The involvement of the styloid process in head and neck pain: a preliminary study. J Oral Rehabil, 27: 275-287.

22. Piagkou M, Anagnostopoulou S, Kouladouros K, Piagkos G (2009) Eagle's syndrome: a review of the literature. Clin Anat, 22: 545-558.

23. Satyapal KS, Kalideen JM (2000) Bilateral styloid chain ossification: case report. Surg Radiol Anat, 22: 211-212.

24. Wang Z, Liu Q, Cui Y, Gao Q, Liu L (2006) Clinical evaluation of the styloid process by plain radiographs and three-dimensional computed tomography. Lin Chuang Er Bi Yan Hou Ke Za Zhi, 20: 60-63.

25. Yuca K, Avcu S, Kiroglu AF, Etlik O, Cankaya H, Kiris M (2010) The detection of Eagle's syndrome with three-dimensional multidetector computerized tomography (CT). Pak J Med Sci, 26: 634-639. 